\title{
LATTICES OF CONTINUOUS MONOTONIC FUNCTIONS
}

\author{
MIRIAM COHEN AND MATATYAHU RUBIN
}

\begin{abstract}
Let $X$ be a compact Hausdorff space equipped with a closed partial ordering. Let $I$ be a linear ordering that either does not have a maximal element or does not have a minimal element. We further assume that $\langle X, I\rangle$ has the Tietze extension property for order preserving continuous functions from $X$ to $I$. Denote by $M(X, I)$ the lattice of order preserving continuous functions from $X$ to $I$. We generalize a theorem of Kaplanski [K], and show that as a lattice alone, $M(X, I)$ characterizes $X$ as an ordered space.
\end{abstract}

Throughout this paper we keep the following notations. An ordered space is a pair $\langle X, \leqslant\rangle$ where $X$ is a compact Hausdorff space, and $\leqslant$ is a partial ordering on $X$ such that $\{\langle x, y\rangle \mid x \leqslant y\}$ is closed in $X \times X$. We abbreviate $\langle X, \leqslant\rangle$ and denote it by $X$ alone.

$I$ denotes a linear ordering: $I$ is always regarded as a topological space with its interval topology. $\bar{I}$ denotes the Dedekind completion of $I$, namely the elements of $\bar{I}$ are those of $I$, and in addition, all pairs $\langle L, R\rangle$ where $L \cup R=I, L \cap R=\varnothing$, if $a \in L$ and $b \leqslant a$ then $b \in L$, and neither does $L$ have a maximum nor does $R$ have a minimum.

A function $f$ from a partially ordered set $\langle A, \leqslant\rangle$ to a partially ordered set $\langle B, \leqslant\rangle$ is order preserving (OP), if for every $a, b \in A$ : if $a \leqslant b$, then $f(a) \leqslant f(b)$. $M(X, I)$ denotes the lattice of OP continuous functions from $X$ to $I$. The lattice operations $\wedge, \vee$ are respectively the pointwise minimum and the pointwise maximum; and $f \leqslant g$ means that for every $x \in X, f(x) \leqslant g(x)$.

We say that $\langle X, I\rangle$ has the Tietze extension property (TEP), if for every closed subset $F$ of $X$ and every OP continuous function $\tilde{f}: F \rightarrow I$ there is $f \in M(X, I)$ which extends $\tilde{f}$.

Nachbin [N, Theorem 6] has shown that if $I=$ Reals, then $\langle X, I\rangle$ has the TEP for every ordered space $X$.

THEOREM 1. For $i=1,2$, let $\left\langle X_{i}, I_{i}\right\rangle$ have the TEP, and assume either $I_{i}$ has no maximal element, or no minimal element. Then, if $M\left(X_{1}, I_{1}\right), M\left(X_{2}, I_{2}\right)$ are lattice isomorphic, then there exists an order preserving homeomorphism between $X_{1}$ and $X_{2}$.

Theorem 1 generalizes [K] where $X$ is assumed to have the trivial partial ordering, namely, every element is comparable just with itself.

Our method of proof is very similar to Kaplansky's. As in the case of the lattice of all continuous functions, there is a natural way to associate every prime ideal in

Received by the editors June 8, 1981 and, in revised form, March 9, 1982.

1980 Mathematics Subject Classification. Primary 54F05, 54C35; Secondary 54C20.

(C)1982 American Mathematical Society 0002-9939/82/0000-U310/\$02.75 
$M(X, I)$ with a point of $X$. Our first goal will be to define the equivalence relation: " $P$ and $Q$ are prime ideals associated with the same point", using only lattice theoretic notions. So, equivalence classes of prime ideals will represent the points of $X$. However, both definitions of when $P$ and $Q$ are associated with the same point and when $x \in \operatorname{cl}(A)$ are more complex than in Kaplansky's case.

We fix a pair $\langle X, I\rangle$ that has the TEP, and show how to interpret points and closed sets in $X$ in terms of the lattice $M(X, I)=M$.

Definition 2. $P \subseteq M$ is an ideal in $M$ if $\varnothing \neq P \neq M$; for every $f \in P$ and $g \leqslant f$, $g \in P$; and for every $f, g \in P, f \vee g \in P$. Note that an ideal means a proper ideal. An ideal $P$ is prime, if for every $f, g \in M$ : if $f \wedge g \in P$, then either $f \in P$ or $g \in P$.

Henceforth, prime ideals will be denoted by $P, Q$ or $R$. Note at this point that being prime can be expressed in lattice theoretic terms only. The following definition in which we associate prime ideals with points of $X$ is slightly weaker than Kaplansky's (see Lemma 4.c).

Definition 3. Let $P$ be a prime ideal of $M$ and $x \in X$. We say that $P$ is associated with $x$ if for every $f \in P, g \in M$ and an open set $U$ containing $x$, if $g(y) \leqslant f(y)$ for all $y \in U$, then $g \in P$.

The proof of the following lemma is similar to the one given in $[\mathbf{K}]$ :

LEMMA 4. (a) If $I \supseteq J$ are ideals, $I$ is associated with $x$ and $J$ is associated with $y$, then $x \leqslant y$. In particular, every proper ideal is associated with at most one point.

(b) Every prime ideal is associated with a unique point.

(c) If $P$ is associated with $x, f \in P$ and $g(x)<f(x)$, then $g \in P$.

Proof. (a) Suppose by contradiction $x \neq y$. Let $f \in J$ and $g \notin I$. There are closed neighborhoods $F$ and $K$ of $x$ and $y$ respectively such that for all $u \in F$ and $v \in K$, $u \neq v$. Let

$$
\tilde{h}=g \uparrow F \cup(F \wedge g) \uparrow K .
$$

Clearly $\tilde{h}$ is OP and continuous so there is $h \in M$ such that $\tilde{h} \subseteq h$. But $h$ must belong to $J$, since $h \leqslant f$ on the neighborhood $K$ of $y$; however, $h$ cannot belong to $I$ since $g \leqslant h$ on the neighborhood $F$ of $x$, and $g \notin I$. This contradicts the fact that $J \subseteq I$.

(b) Suppose $P$ is a prime ideal associated to no point of $X$. Then for every $x \in X$, we have functions $f, g$ and an open $U \ni x$, such that $g(y) \leqslant f(y)$ for all $y \in U$, but $f \in P$ and $g \notin P$. A finite number of these neighborhoods cover $X$. Let $f_{1}, \ldots, f_{n}$ and $g_{1}, \ldots, g_{n}$ be the corresponding functions, and define $h=f_{1} \vee \cdots \vee f_{n}$ and $k=g_{1}$ $\wedge \cdots \wedge g_{n}$. Then $k \leqslant h$, implying $k \in P$, however $k \notin P$ by primeness of $P$, a contradiction.

(c) Follows from continuity.

Now, for any prime ideal $P$, denote by $x_{P}$ the point with which it is associated, and define $\alpha_{P} \in \bar{I}$ by $\alpha_{P}=\sup \{f(x) \mid f \in P\}$. Let $[x]=\left\{P \mid x_{P}=x\right\}$.

The following prime ideals will turn out to be of importance.

Let $x \in X, \alpha$ be a nonminimal of $I$ and $\beta$ be a nonmaximal element of $I$. Let $P_{x}^{<\alpha}=\{f \in M \mid f(x)<\alpha\}$, and $P^{<\beta}=\{f \in M \mid f(x) \leqslant \beta\}$. These are prime ideals associated with $x$, and every prime ideal $Q$, with $\alpha=\alpha_{Q}$ and $x=x_{Q}$, satisfies: $P_{x}^{<\alpha} \subseteq Q \subseteq P_{x}^{<\alpha}$. 
Notice that $x<y$ implies $P_{x}^{<\alpha} \supseteq P_{y}^{<\alpha}$, and therefore, unlike in [K], $P \supseteq Q$ does not imply that $x_{P}=x_{Q}$. However, we have the following proposition:

Proposition 5. If $P \supseteq Q$, then (1) $x_{P} \leqslant x_{Q}$ and (2) $\alpha_{P} \geqslant \alpha_{Q}$.

Proof. (1) is just a reformulation of Lemma 4.a.

(2) Suppose $\alpha_{P}<\alpha_{Q}$. Obviously this is impossible if $x_{P}=x_{Q}$. So assume $x_{P}<x_{Q}$. If there exists an $\alpha$ such that $\alpha_{P}<\alpha<\alpha_{Q}$, then let $g=\alpha$ be the constant function $\alpha$. Then $g \in Q-P$, a contradiction. If there does not exist such an $\alpha$, there exists $f \in Q$ with $f\left(x_{Q}\right)=\alpha_{Q}$. Let $U=\left\{y \mid f(y)>\alpha_{P}\right\}=\left\{y \mid f(y) \geqslant \alpha_{Q}\right\}$. Then $U$ is both open and closed, $x_{Q} \in U$ and $x_{P} \notin U$. Define $\tilde{g}=f \wedge U \cup \alpha_{Q} \uparrow x_{P}$, and let $g$ be the extension of $\tilde{g}$ to an element of $M$. Since $g=f$ on $U$, we have $g \in Q$. But since $g\left(x_{P}\right)>\alpha_{P}, g \notin P$, a contradiction.

CoRollary 6. (a) If $P \supseteq Q \supseteq R$ and $x_{P}=x_{R}$, then $x_{P}=x_{Q}$.

(b) $P_{x}^{\leqslant \alpha} \supseteq Q$ if and only if $x \leqslant x_{Q}$ and $\alpha \geqslant \alpha_{Q}$.

(c) $Q \supseteq P_{x}^{<\alpha}$ if and only if $x_{Q} \leqslant x$ and $\alpha_{Q} \geqslant \alpha$.

Proof. (b) Assume $x \leqslant x_{Q}$ and $\alpha \geqslant \alpha_{Q}$, and let $f \in Q$. Then $f(x) \leqslant f\left(x_{Q}\right) \leqslant \alpha_{Q}$ $\leqslant \alpha$, thus $f \in P_{x}^{\leqslant \alpha}$. The proof of (c) is similar, and the rest is a corollary to Proposition 5.

In Proposition 10 we shall give a lattice theoretic criterion for two prime ideals to be associated to the same point. In the following definitions we distinguish between the possibilities of having either no minimal element or no maximal element. The latter will be denoted by *. Observe that $I$ has no minimal (maximal) element iff $M$ has no minimal (maximal) element.

Let $P_{1} P_{2}=\left\{P^{\prime}\right.$ prime $\left.\mid P_{1} \supseteq P^{\prime} \supseteq P_{2}\right\}$.

Definition 7. (1) Let $S(P, Q)$ denote the following property: (a) $P \supseteq Q$; (b) for every $f \in M$ there is a prime $R$ such that $f \notin R, R \subseteq Q$ and $P R=P Q \cup Q R$.

(2) Let $S^{*}(P, Q)$ denote the following property: (a) $P \supseteq Q$; (b) for every $f \in M$ there is a prime $R$ such that: $f \in R, R \supseteq P$ and $R Q=R P \cup P Q$.

Lemma 8. (1) Suppose $M$ does not have a minimal element, then if $x_{P}=x$ and $\alpha_{P}>\alpha$, then $S\left(P, P_{x}^{<\alpha}\right)$ holds.

(2) Suppose $M$ does not have a maximal element, then if $x_{P}=x$ and and $\dot{\alpha}_{P}<\alpha$, then $S^{*}\left(P_{x}^{<\alpha}, P\right)$ holds.

Proof. (1) (a) of $S(P, Q)$ follows from the definitions. (b) Let $f \in M$. If $f(x) \geqslant \alpha$, take $R=P_{x}^{<\alpha}$. If $f(x)<\alpha$, let $R=P_{x}^{<f(x)}$, then $P_{x}^{<\alpha} \supseteq R$. Furthermore, let $T \in P R$; then by Corollary 6.a, $x_{T}=x$, and by Proposition 5, $f(x) \leqslant \alpha_{T}<\alpha_{P}$. If $\alpha_{T}<\alpha$, then $P_{x}^{<\alpha} \supseteq T$, and if $\alpha_{T} \geqslant \alpha, T \supseteq P_{x}^{<\alpha}$. Hence $P R \subseteq P P_{x}^{<\alpha} \cup P_{x}^{<\alpha} R$.

(2) is proved analogously.

LEMma 9. (1) Suppose $M$ has no minimal elements, and $P, Q$ satisfy $S(P, Q)$, then $x_{P}=x_{Q}$. (2) Suppose $M$ has no maximal element, and $P, Q$ satisfy $S^{*}(P, Q)$, then $x_{P}=x_{Q}$.

Proof. (1) Assume $x_{P} \neq x_{Q}$. By Proposition 5, $x_{P}<x_{Q}$ and $\alpha_{P} \geqslant \alpha_{Q}$. Let $\varepsilon<\delta<\alpha_{Q}$, and let $f$ be the constant function with value $\varepsilon$. 
By $S(P, Q)$ there exists a prime $R$ such that $Q \supseteq R \boxplus f$, and $P R=P Q \cup Q R$. Since $f \notin R, \alpha_{R} \leqslant \delta$; and since $Q \supseteq R, x_{Q} \leqslant x_{R}$. By Corollary 6, $P \supseteq P_{x_{P}}^{<\delta} \supseteq R$; that is: $P_{x_{P}}^{<\delta} \in P R$. However, $Q \supseteq P_{x_{P}}^{<\delta}$ is impossible since $x_{Q}>x_{P}$, and $P_{x_{P}}^{<\delta} \supseteq Q$ is impossible since $\delta<\alpha_{Q}$, contradicting $S(P, Q)$.

(2) is proved analogously.

We are ready to give the following criterion:

Proposition 10. (1) Assume $M$ has no minimal element. Then $x_{P}=x_{Q}$ if and only if there exists a prime ideal $R$ such that both $S(P, R)$ and $S(Q, R)$ hold. (2) Assume $M$ has no maximal element. Then $x_{P}=x_{Q}$ if and only if there exists a prime $R$ such that both $S^{*}(R, P)$ and $S^{*}(R, Q)$ hold.

Proof. (1) The sufficiency follows from Lemma 9. Conversely, assume $x_{P}=x_{Q}=$ $x$, and let $\beta<\min \left\{\alpha_{P}, \alpha_{Q}\right\}$. By Lemma 8 , both $S\left(P, P_{x}^{<\beta}\right)$ and $S\left(Q, P_{x}^{<\beta}\right)$ hold.

(2) is proved analogously.

We proceed with

Definition 11. Let $f, g \in M$ and $x \in X$, we say $f \equiv_{x} g$ if for every $P \in[x]$, $g \in P \Leftrightarrow f \in P$.

Note that by Proposition 10, the notion of $f \equiv_{x} g$ can be expressed in lattice theoretic terms.

The following series of lemmas culminates in Proposition 16 which gives a necessary and sufficient condition for $f \equiv_{x} g$.

Definition 12. An ultrafilter in $X$ is a maximal set of open sets, having the finite intersection property.

It is well known that every set of open sets with the finite intersection property is contained in an ultrafilter. Assume $U$ is an open subset of $X$ and $x \in \operatorname{cl}(U)$. Let $L=\{U\} \cup\{W \mid W$ is an open neighbourhood of $x\}$. Then $L$ has the finite intersection property. Let $D$ denote an ultrafilter containing $L$.

Lemma 13. Let $x, U$, and $D$ be as above, and $g \in M$. Let $Q=\{h \in M \mid$ thre exists $W \in D$ with $h(w) \leqslant g(w)$ for all $w \in W\}$. Then, $Q$ is a prime ideal associatd with $x$.

Before proving the lemma we need some preliminaries.

Lemma 14. Let $D$ be an ultra-filter in $X$, and $S, T$ open sets such that: $\operatorname{int}(\mathrm{cl}(S))=$ $\operatorname{int}(\operatorname{cl}(T))$, then $S \in D$ if and only if $T \in D$.

Proof. Assume $T \notin D$. Then there exists some $W \in D$ such that $T \cap W=\varnothing$. If $z \in \operatorname{cl}(T) \cap W$, then every open neighbourhood of $z$ intersects $T$ nontrivially. Since $W$ is such a neighbourhood we get a contradiction. Thus $\operatorname{cl}(T) \cap W=\varnothing$, but then $\operatorname{int}(\operatorname{cl}(T)) \cap W=\varnothing$. This implies that $\varnothing=\operatorname{int}(\operatorname{cl}(S)) \cap W \supseteq S \cap W$. Hence $S \notin$ D.

Lemma 15. Let $D$ be an ultrafilter in $X$ and let $F_{1}, F_{2}$ be closed subsets of $X$ such that $F_{1} \cup F_{2} \supseteq W \in D$. Then either $F_{1}$ or $F_{2}$ contain an element of $D$.

Proof. Without loss of generality, assume $W=\operatorname{int}\left(F_{1} \cup F_{2}\right)$. Let $W_{i}=\operatorname{int}\left(F_{i}\right)$, $i=1,2$. We will show that either $W_{1}$ or $W_{2}$ are elements of $D$. Assume the contrary. 
Then there exist $V_{1}, V_{2} \in D$ such that $W_{i} \cap V_{i}=\varnothing, i=1,2$. Hence $\left(V_{1} \cap V_{2}\right) \cap$ $\left(W_{1} \cup W_{2}\right)=\varnothing$. This implies that $W_{1} \cup W_{2} \notin D$. However, $W=\operatorname{int}(\operatorname{cl}(W))=$ $\operatorname{int}\left(\operatorname{cl}\left(W_{1} \cup W_{2}\right)\right)$, and $W \in D$, contradicting Lemma 14 .

Let us prove now Lemma 13. Let $x, U, D, g$ and $Q$ be as in Lemma 13. Then obviously $Q$ is an ideal. Assume $f_{1} \wedge f_{2} \in Q$ and let $F=\left\{z \in X \mid\left(f_{1} \wedge f_{2}\right)(z) \leqslant\right.$ $g(z)\}$ and $F_{i}=\left\{z \in X \mid f_{i}(z) \leqslant g(z)\right\}, i=1,2$. Then $F=F_{1} \cup F_{2}$. Since $f_{1} \wedge f_{2} \in Q$, there exists $W \in D$ with $W \subseteq F$. Hence by Lemma 15 either $F_{1}$ or $F_{2}$ contains an element of $D$. This implies that either $f_{1}$ or $f_{2}$ are in $Q$. Thus $Q$ is prime. Let $h \in Q$ and assume $f(v) \leqslant h(v)$ for all $v$ in some open neighbourhood $V$ of $x$. Since $h \in Q$, there is some $W \in D$ s.t. $h(w) \leqslant g(w)$ for all $w \in W$. Hence, for $w \in V \cap W$, $f(w) \leqslant y(w)$. Since $V \cap W \in D$, this implies that $f \in Q$. We have shown that $Q$ is assoicated with $x$.

Proposition 16. Let $f, g \in M$, and $x \in X$. Then $f \equiv_{x} g$ if and only if there exists an open neighbourhood $W$ of $x$, such that $f \uparrow W=g \uparrow W$.

Proof. Assume $f \equiv_{x} g$, but in each open neighbourhood of $x$ there exists a $y$ with $f(y) \neq g(y)$. Let $U=\{y \mid g(y)<f(y)\}$; without loss of generality, $x \in \operatorname{cl}(U)$. Let $D$ be an ultrafilter containing $U$ and all the open neighbourhoods of $x$. Let $Q=(h \in M \mid$ for some $V \in D, h(v) \leqslant g(v)$ for all $v \in V)$. Then by Lemma $13, Q$ is a prime ideal associated to $x$. However, $g \in Q$ while $f \notin Q$, contradicting $f \equiv_{x} g$.

The converse is obvious by definition.

In Theorem 18 we show how to describe the closed sets of $X$ in terms of the lattice $M$ and thus get the main theorem, Theorem 1, as a corollary.

Definition 17. Let $x \in X$ and $A \subseteq X$. Let $C(A, x)$ mean that

$$
(\exists P \in[x]) \exists f\left(\forall f^{\prime} \equiv_{x} f\right)(\forall g \in P) \exists Q\left(x_{Q} \in A \text { and } f^{\prime} \notin Q \text { and } q \in Q\right) \text {. }
$$

Let $C^{*}(A, x)$ mean that

$$
(\exists P \in[x]) \exists f\left(\forall f^{\prime} \equiv_{x} f\right)(\forall g \notin P) \exists Q\left(x_{Q} \in A \text { and } f^{\prime} \in Q \text { and } g \notin Q\right) \text {. }
$$

THEOREM 18. (1) If $M$ has no minimal element, then for every $A \subseteq X$, and $x \in X$ : $C(A, x)$ iff $x \in \operatorname{cl}(A)$.

(2) If $M$ has no maximal element, then for every $A \subseteq X$ and $x \in X: C^{*}(A, x)$ iff $x \in \operatorname{cl}(A)$.

Proof. (1) Assume $x \in \operatorname{cl}(A)$, let $f \in M$ and $P=P_{x}^{<f(x)}$. Let $f^{\prime} \equiv_{x} f, g \in P$ and let $U$ be an open neighbourhood of $x$ such that $f^{\prime} \uparrow U=f \uparrow U$. There is an open $W$ such that $x \in W \subseteq U$, and for every $w \in W, g(w)<f^{\prime}(w)=f(w)$. Let $z \in A \cap W$ and $Q=P_{z}^{<f^{\prime}(z)}$. Then $g \in Q$ and $f^{\prime} \in Q$.

Suppose $x \notin \operatorname{cl}(A)$; we show that $C(A, x)$ does not hold. We will show that for every $P \in[x]$ and $f \in M$, there is $f^{\prime} \equiv_{x} f$ and $g \in P$ such that for every $a \in A$ there exists a neighbourhood $U$ of $a$ on which $f^{\prime} \leqslant g$. Clearly this will contradict $C(A, x)$.

So let $P \in[x]$ and $f \in M$. Let $F=\{z(\exists a \in \operatorname{cl}(A))(z \leqslant a \leqslant x)\}$. Clearly $F$ is closed [N, p. 44] and for every $z \in F$ and $z_{1} \leqslant z, z_{1} \in F$. Let $K=\{y \mid x \leqslant y\}$. Since $F, K$ are closed, there is an open $U \supseteq K$ such that $\operatorname{cl}(U) \cap F=\varnothing$. Let $\gamma<\delta<$ $\min \left\{\alpha_{P}, f(\operatorname{cl}(U))\right\}$, and let $\tilde{h}=f \uparrow \operatorname{cl}(U) \cup \gamma \uparrow F . \tilde{h}$ is a continuous OP function on 
$\operatorname{cl}(U) \cup F$. Let $f^{\prime} \in M$ be an extension of $\tilde{h}$; then $f^{\prime} \equiv_{x} f$, by Proposition 16 and since $f^{\prime} \uparrow U=f \uparrow U$.

Let $F_{1}=F \cup\{x\}$ and $K_{1}=\left\{z \in \operatorname{cl}(A) \mid f^{\prime}(z) \geqslant \delta\right\}$. Then, if $z \in K_{1}$ and $y \in F_{1}$ then $z \$ y$. For suppose by contradiction that $z \leqslant y$, then by definition, $z \in F$. However, this implies that $f^{\prime}(z)=\gamma<\delta$, a contradiction. By [N, p. 46], there exist an open $V \supseteq K_{1}$, such that for every $v \in \operatorname{cl}(V)$ and $y \in F_{1}, v \$ y$. Let $\tilde{g}_{1}=\delta$ i $F_{1} \cup$ $\left(f^{\prime} \vee \delta\right) \uparrow \operatorname{cl}(V)$. Clearly $\tilde{g}_{1}$ is continuous and OP. Let $g_{1} \in M$ be an extension of $\tilde{g}_{1}$, and let $g=g_{1} \vee \delta$. Now, let $a \in A$; if $f^{\prime}(a)<\delta$ then $f^{\prime}(a)<g(a)$, and hence the inequality holds on a neighbourhood of $a$. If $f^{\prime}(a) \geqslant \delta$, then $a \in K_{1} \subseteq V$, and hence by the definition of $g_{1}$ and $g, g \geqslant f^{\prime}$ on the neighbourhood $V$ of $a$.

Proof of Theorem 1. Let $\varphi: M\left(X_{1}, I_{1}\right) \rightarrow M\left(X_{2}, I_{2}\right)$ be a lattice isomorphism. Clearly, $M\left(X_{1}, I_{1}\right)$ has no minimal element iff $M\left(X_{2}, I_{2}\right)$ does not have a minimal element, and the same holds for the existence of a maximal element. W.l.o.g. $M\left(X_{i}, I_{i}\right)$ do not have a minimal element. $\varphi$ induces a 1-1 correspondence $\tilde{\varphi}$ between $X_{1}$ and $X_{2}$ in the following way: let $x \in X_{1}$ choose a prime ideal $P$ in $M\left(X_{1}, I_{1}\right)$ such that $x_{P}=x$ and define $\tilde{\varphi}(x)=x_{\varphi(P)}$. Since " $x_{P}=x_{Q}$ " is expressible in lattice theoretic terms, $\tilde{\varphi}(x)$ is independent of the choice of $P$, and it is clearly $1-1$ and onto. Let $P$ be a prime ideal in $M\left(X_{1}, I_{1}\right)$ and $\mathscr{P}$ be a set of prime ideals; the notion " $x_{P} \in \operatorname{cl}\left(\left\{x_{Q} \mid Q \in \mathcal{P}\right\}\right)$ " is expressible in lattice theoretic terms; this implies that $\tilde{\varphi}$ is a homeomorphism. A similar argument shows that $\tilde{\varphi}$ is OP. Q.E.D.

Discussion and open questions. There were three possible strengthenings of Theorem 1 , that we considered.

(1) Can the linear ordering $I$ in Theorem 1 be replaced by a general topological lattice without a minimum or a maximum?

The answer to this question is no. Let $X, Y$ be metric compact spaces, then the set $C(Y, \mathbf{R})$ of all continuous functions from $Y$ to $\mathbf{R}$ with the usual maximum topology is a topological lattice. Consider now the following pairs $\langle X \times Y, \mathbf{R}\rangle$ and $\langle X, C(Y, \mathbf{R})\rangle$. Both pairs have the TEP.

$$
M(X \times Y, \mathbf{R}) \stackrel{\varphi}{\cong} M(X, C(Y, \mathbf{R})), \quad \text { where } \varphi(f)(x)(y) \stackrel{\text { def }}{=} f(x, y) .
$$

However, $X$ and $Y$ can be chosen so that $X \times Y$ and $X$ are nonhomeomorphic.

One can still ask whether what we have presented is more or less the only kind of counterexamples. A more definite question is whether $M\left(X_{1}, L_{1}\right) \cong M\left(X_{2}, L_{2}\right)$ implies that the two lattices are homeomorphic in the compact open topology.

(2) Was it necessary in Theorem 1 to impose the condition that $I$ does not have both a minimum and a maximum?

The above condition is necessary. We give a counterexample. Let $I_{n}$ denote a linear ordering with $n$ elements ( $n$ is finite). $I_{n}$ is a compact Hausdorff ordered space when equipped with its interval topology, and the pair $\left\langle I_{n}, I_{k}\right\rangle$ has the TEP.

PROPOSITION. If $k \geqslant 1$ and $n \geqslant 2$, then $M\left(I_{k}, I_{n}\right) \cong M\left(I_{n-1}, I_{k+1}\right)$.

Proof. W.l.o.g. $I_{m}=\{1, \ldots, m\}$. Let $I^{*}$ denote the reverse ordering of $I$. We define $\varphi: M\left(I_{k}, I_{n}\right) \rightarrow M\left(I_{n-1}^{*}, I_{k+1}^{*}\right):$ if $f \in M\left(I_{k}, I_{n}\right)$ and $j \in I_{n-1}$, then $\varphi(f)(j)$ $=1+$ number of $i$ such that $f(i) \leqslant j$. It is easy to check that $\varphi$ is an isomorphism between the above lattices. 
It is still conceivable that it is possible to characterize all pairs $\left\langle X_{1}, I_{1}\right\rangle,\left\langle X_{2}, I_{2}\right\rangle$ that have the TEP and satisfy $M\left(X_{1}, I_{1}\right) \cong M\left(X_{2}, I_{2}\right)$.

We conjecture that if $\left\langle X_{i}, I_{i}\right\rangle$ have the TEP and $M\left(X_{1}, I_{1}\right) \cong M\left(X_{2}, I_{2}\right) \cong$ $M\left(X_{3}, I_{3}\right)$, then for some $i \neq j, X_{i}$ and $X_{j}$ are homeomorphic.

(3) In both $[\mathrm{K}]$ and this work, it remains unsettled whether $M(X, I)$ determines $I$. Question. Suppose that $\left\langle X_{i}, I_{i}\right\rangle$ have the TEP and $I_{i}$ has either no maximum or no minimum, $i=1,2$, and suppose further that $M\left(X_{1}, I_{1}\right) \cong M\left(X_{2}, I_{2}\right)$. Does it follow that $I_{1} \cong I_{2}$ ?

Observation. The answer of the above question is positive, if $X_{1}$ has a maximum. It is easy to see that for every $f \in M(X, I)$ the ideal $P_{f}$ def $=\{g \mid g \leqslant f\}$ is prime iff $X$ has a maximum, and $f$ is a constant function. Suppose $X_{1}$ has a maximum and $M\left(X_{2}, I_{2}\right) \stackrel{\varphi}{\cong} M\left(X_{1}, I_{1}\right)$, hence $X_{2}$ has a maximum. Clearly, $f \in M\left(X_{1}, I_{1}\right)$ is constant iff $\varphi(f)$ is. Hence $I_{1} \cong I_{2}$.

\section{REFERENCES}

[K] I. Kaplansky, Lattices of continuous functions, Bull. Amer. Math. Soc. 39 (1947), 617-623.

[N] L. Nachbin, Topology and order, Van Nostrand, Princeton, N. J., 1965.

Department of Mathematics, Ben Gurion University of the Negev, BeER Sheva, ISRael 